\title{
Extracellular vesicles from bone marrow-derived multipotent mesenchymal stromal cells regulate inflammation and enhance tendon healing
}

\author{
Zhengzhou Shi, Qi Wang and Dapeng Jiang*
}

\begin{abstract}
Background: Extracellular vesicles from bone marrow-derived multipotent mesenchymal stromal cells (BMSC-EVs) can play important roles in the repair of injured tissues. However, no reports have investigated the role and underlying mechanisms of BMSCs-EVs in the tendon repair process. We hypothesized that BMSC-EVs may play a role in modulating inflammation during tendon healing and improving tendon repair in a rat model of patellar tendon injury.

Methods: First, we created window defects in the patellar tendons of Sprague-Dawley rats. Rats $(n=16)$ were then randomly assigned to three groups: BMSC-EVs group, Fibrin group, and control group. Rats in the BMSC-EVs group were treated with BMSC-EVs and fibrin glue $(25 \mu \mathrm{g}$ in $10 \mu \mathrm{L})$. Rats in the fibrin group were treated with fibrin only, and those in the control group received no treatment. Histopathology, immunohistochemistry, and gene expression analyses were performed at 2 and 4 weeks after surgery.

Results: At 4 weeks, tendons treated with BMSC-EVs showed regularly aligned and compact collagen fibers as compared with the disrupted scar-like healing in rats in the fibrin and control groups. The expression of genes related to tendon matrix formation and tenogenic differentiation: collagen (COL)-1a1, scleraxis (SCX), and tenomodulin (TNMD) was significantly higher in the BMSC-EVs group than in the other two groups. With histopathology, we observed significantly higher numbers of CD146+ tendon stem cells and fewer numbers of apoptotic cells and C-C chemokine receptor type 7 (CCR7)-positive proinflammatory macrophages in the BMSC-EVs group. BMSC-EVs treatment also led to an increase in the expression of anti-inflammatory mediators (IL-10 and IL-4) at 2 weeks after surgery.
\end{abstract}

Conclusions: Overall, our findings show that the local administration of BMSC-EVs promotes tendon healing by suppressing inflammation and apoptotic cell accumulation and increasing the proportion of tendon-resident stem/ progenitor cells. These findings provide a basis for the potential clinical use of BMSC-EVs in tendon repair.

Keywords: Mesenchymal stem cells, Extracellular vesicles, Macrophages, Tendon stem cells, Tendon healing

\section{Background}

Tendons play key roles in connecting muscles to bones. They are frequently injured in both occupational and athletic activities. Tendon injuries are an acute health care burden and have become a significant challenge in orthopedics [1]. Natural tendon healing is a complex

*Correspondence: jdp509@163.com

Department of Urology, Shanghai Children's Medical Center, Shanghai Jiao Tong University School of Medicine, Shanghai 200127, China process consisting of three overlapping stages: inflammation, proliferation, and remodeling. Tendon healing is slow compared with other types of connective tissue healing because of its poor vascularization and the excessive load-bearing it must withstand [2]. A previous study showed that inflammatory factors are dramatically upregulated within the first 7 days after tendon injury [3]. An intense early inflammatory cascade often results in the formation of a scar-like tendon, and is associated with chronic matrix degradation and the formation of 
adhesions, which impede the intrinsic repair process and lead to a tendon with poor tissue quality and inferior mechanical properties [4]. In recent years, tissue engineering and other biological-based therapeutic strategies have been used to modulate inflammation at the repair site to effectively enhance tendon healing [5-7].

The use of mesenchymal stem cells (MSCs) is currently an attractive solution for tendon repair and regeneration. Recent findings show that adipose tissue-derived mesenchymal stromal cells and tendon stem/progenitor cells (TSCs) can modulate the inflammatory environment by regulating the activities of resident macrophages to enhance tendon healing $[8,9]$. Indeed, one study showed that bone marrow-derived multipotent mesenchymal stromal cells (BMSCs) can improve early tendon healing both histologically and biomechanically [10]. However, the implantation of BMSCs can also result in ectopic bone formation in the tendon or contribute to teratoma formation [11, 12]. Recent studies have demonstrated that MSC transplantation therapy promotes tissue repair mainly through a paracrine mechanism [13-15], and that EVs play an important role in the function of MSCs $[16,17]$. More recently published results indicate that EVs derived from BMSCs (BMSCs-EVs) have promise in attenuating inflammation and apoptosis during tissue repair [18].

We hypothesized that BMSC-EVs may also play a role in modulating inflammation during tendon healing. To test this hypothesis, EVs were isolated from BMSCs and delivered to a rat model of tendon repair. The impact of BMSC-EVs on the expression of proinflammatory cytokines, anti-inflammatory mediators, and macrophages during tendon healing was investigated in vivo. The effect of BMSC-EVs on the quality of the repaired tendon tissue was also characterized.

\section{Methods}

\section{Isolation and culture of rat BMSCs}

Bone marrow-derived multipotent mesenchymal stromal cells were isolated from eight Sprague-Dawley rats. Animal experiments were performed according to the Rules and Regulations of the Animal Care and Use Committee at our University. BMSCs were isolated from rat bone marrow as described previously [10]. Briefly, bone marrow was flushed from the bone marrow cavities, collected into centrifuge tubes, and mononuclear cells were isolated by Ficoll density gradient. The mononuclear cells were then suspended in alpha-modified Eagle's medium $(\alpha-$ MEM) containing $10 \%$ fetal bovine serum (FBS) and plated into T-75 flasks. Cells were incubated at $37{ }^{\circ} \mathrm{C}$ with $5 \% \mathrm{CO}_{2}$, with the medium changed every 3 days. Nonadherent cells were discarded after $48 \mathrm{~h}$. The bone marrow cells at the third passage were harvested and characterized. BMSCs were identified by flow cytometry with a fluorescence-labeled antibody for the positive surface markers CD44 and CD90. The negative surface markers: CD11b and CD34 were investigated as negative marker to exclude hematopoietic lineages. Moreover, morphology and multipotency of BMSCs were measured. The BMSCs used in this study were between passage 3 and 5 .

\section{Isolation and identification of BMSCs-EVs}

At $80 \%$ to $90 \%$ confluence, BMSCs were rinsed with phosphate-buffered saline (PBS) and cultured in Mesen Gro MSC medium (Thermo Fisher Scientific) for an additional $48 \mathrm{~h}$. Conditioned media was collected and EVs were isolated, as described previously [16]. The purification of BMSCs-EVs involves several centrifugation and ultracentrifugation. The conditioned media was centrifuged sequentially at $300 \times g$ for 10 min followed by $2000 \times g$ for $10 \mathrm{~min}$ to remove cellular debris. The supernatants were then ultracentrifuged at $100,000 \times g$ for $2 \mathrm{~h}$ to obtain a pellet containing the EVs, which was resuspended in $200 \mu \mathrm{L}$ of PBS. EVs-enriched fraction was centrifuged at $1500 \mathrm{~g}, 30 \mathrm{~min}$ with $100-\mathrm{kDa}$ molecular weight cutoff (MWCO) hollow fiber membrane (Millipore, Billerica, MA, USA). Then, EVs were passed through a 0.22$\mu \mathrm{m}$ filter. The total protein concentration in the EVs was quantitated using the Micro Bicinchoninic Acid (BCA) Protein Assay Kit (Pierce) following the manufacturer's instructions. Transmission electron microscopy (TEM) and western blotting were used to examine the morphology and the quality of the EVs. The size of EVs were analyzed with use of qNano. EVs were stored at $4{ }^{\circ} \mathrm{C}$ for no more than $1 \mathrm{~h}$ before used for experiments.

\section{Surgical procedure and treatment}

A patellar tendon injury model was created in SpragueDawley rats, according to a previously published method [7]. Briefly, in 48 rats, the central one-third of the patellar tendon was removed from the distal apex of the patellar to the insertion of the tibial tuberosity to create a tendon defect (Fig. 1). Then, rats were allocated to one of three groups $(n=16)$ : BMSC-EVs group, treated with $10 \mu \mathrm{L}$ of fibrin containing $25 \mu \mathrm{g}$ BMSC-EVs; Fibrin group, treated with $10 \mu \mathrm{L}$ of fibrin glue alone; and the control group, which was left untreated. Fibrin glue containing EVs was placed in the window defect of patellar tendon after rat tendon injury model. The fibrin glue $\left(\right.$ Baxter $^{\circledR}$, Vienna, Austria) was considered to act as a useful vehicle of growth factors or cells. It has been used extensively in all kinds of surgery and research [19, 20]. Fibrin solution consists of two main components: fibrinogen and thrombin. The final concentration of fibrinogen and thrombin was $80 \mathrm{mg} / \mathrm{mL}$ and 600 units $/ \mathrm{mL}$, respectively. It could 


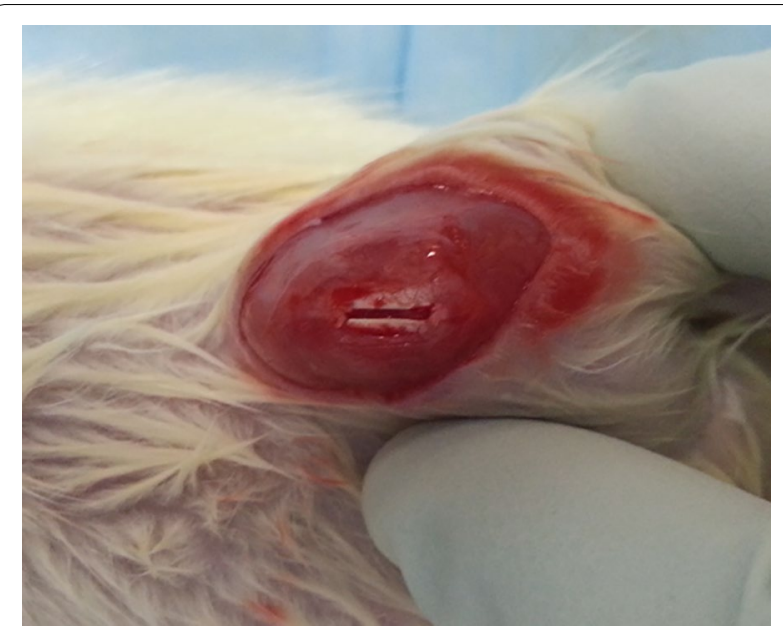

Fig. 1 Patellar tendon injury model. The central one-third of the patellar tendon was removed to create a tendon defect

produce a fibrin clot in about $10 \mathrm{~s}$ after applied to injury site. Mixing EVs lysates $(25 \mu \mathrm{g})$ in fibrin glue $(10 \mu \mathrm{L})$ is easy to deliver locally in window defect of patellar tendon.

At 2 and 4 weeks after surgery, eight rats in each group were killed, and the tissues surrounding the injured patellar tendons were harvested for histology, immunohistochemistry, and gene expression analysis.

\section{Histology and immunohistochemistry}

Rat patellar tendon tissues were harvested at 4 weeks and fixed in $4 \%$ formaldehyde solution and embedded in paraffin. Samples were sectioned longitudinally at a thickness of $5 \mu \mathrm{m}$, and then processed for histological examination using hematoxylin-eosin (H\&E) and Masson's trichrome staining. The organization of fibrous connective tissue within the defect site was evaluated using a parallel fiber alignment scoring method [21], as previously described: $3=75 \%$ to $100 \%$ parallel fiber alignment; $2=50 \%$ to $75 \%$ parallel fiber alignment; $1=25 \%$ to $50 \%$ parallel fiber alignment; and $0=0 \%$ to $25 \%$ parallel fiber alignment. All sections were analyzed by a single pathologist, who was blinded of the treatment groups.

Specimens harvested at 2 weeks were used for immunohistochemistry to examine expression changes in CD146, cleaved caspase-3, CD163, C-C chemokine receptor type 7 (CCR7), interleukin (IL)-10, and IL-6 (all from Abcam; 1:100 dilution). Immunohistochemical staining with anti-rat type I collagen antibody (1:300; \#sc-25974, Santa Cruz Biotechnology, Dallas, TX) was also performed to evaluate tendon healing. Briefly, nonspecific reactive sites were masked with $5 \%$ bovine serum albumin before the slides were incubated overnight at $4{ }^{\circ} \mathrm{C}$ with primary antibodies at $1: 100$ or $1: 300$ dilution, as specified. Slides were then incubated with a species appropriate secondary antibody in the dark at room temperature for $60 \mathrm{~min}$. Nuclei were counterstained with Hoechst fluorochrome 33342 (1 mg/mL; Sigma-Aldrich, St. Louis, MO, \#B2261). Positive cells in the healing tendon tissue were quantified using light microscopy at the magnification of $400 \times$, defined by an ocular morphometric grid. For each animal, 15 randomly selected tissue sections were analyzed.

\section{Gene expression}

Gene expression changes in the healing tendons were determined using real time PCR. Rats were anesthetized with isoflurane gas on day 14 after surgery. 8 healing patellar tendon tissues in each group were processed for mRNA extraction. Total RNA was extracted using an RNeasy mini kit (Qiagen; Hilden, Germany). cDNA was synthesized using the First-strand kit (Invitrogen; Carlsbad, CA). qRT-PCR was carried out with the QuantiTect SYBR Green RT-PCR kit (Qiagen). Total RNA isolation, cDNA synthesis, and gene expression assay were performed as described previously [22]. Relative gene expression levels were calculated with the $2^{\Delta \mathrm{CT}}$ formula. The gene expression levels were normalized with respect to the control group. Each gene analysis was performed in triplicate.

Rat-specific primers were synthesized by Invitrogen (Carlsbad, CA) and used for tenomodulin (TNMD), scleraxis (SCX), collagen type I, collagen type III, IL-1B, IL-6, interferon gamma (IFNY), IL-4, IL-10, IL-13, CCR7, and CD163 testing. Glyceraldehyde-3-phosphate dehydrogenase (GAPDH) was used as an internal control (Table 1).

\section{Isolation and culture of rat tendon cells}

Patellar tendons from SD male rats were used as explants for primary cell cultures. Patellar tendons were cut into small sections, and then placed and cultured in 6-well culture plates in aseptic conditions. After 5 min of airdrying for better adherence, Dulbecco's Modified Eagle Medium containing 20\% fetal bovine serum and $1 \%$ penicillin/streptomycin were supplemented to each well. After 3-4 days in culture, cells began to emerge from the tendon pieces. After the cells reached $80 \%$ confluence, tendon cells were detached with trypsin and mixed together as passage 0 . Tendon cells at passages 3 were used in the following experiments. 
Table 1 Rat primers used for qRT-PCR analysis

\begin{tabular}{|c|c|}
\hline Gene & Primer sequence \\
\hline TNMD & $\begin{array}{l}\text { F: 5'-CCATGCTGGATGAGAGAGGTTAC-3' } \\
\text { R: 5'-CACAGACCCTGCGGCAGTA-3' }\end{array}$ \\
\hline $\operatorname{scx}$ & $\begin{array}{l}\text { F: 5'-AACACGGCCTTCACTGCGCTG-3' } \\
\text { R: 5'-CAGTAGCACGTTGCCCAGGTG-3' }\end{array}$ \\
\hline COL1a1 & $\begin{array}{l}\text { F: 5'-CCGGACTGTGAGGTTAGGAT-3' } \\
\text { R: 5'-AACCCAAAGGACCCAAATAC-3' }\end{array}$ \\
\hline COL3a1 & $\begin{array}{l}\text { F: 5'-AACGGAGCTCCTGGCCCCAT-3' } \\
\text { R: 5'-ATTGCCTCGAGCACCTGCGG-3' }\end{array}$ \\
\hline$I L-1 B$ & $\begin{array}{l}\text { F: 5'-AGCAGCTTTCGACAGTGAGG-3' } \\
\text { R: 5'-CTCCACGGGCAAGACATAGG-3' }\end{array}$ \\
\hline IL-6 & $\begin{array}{l}\text { F: 5'-AGAAAAGAGTTGTGCAATGGCA-3' } \\
\text { R: 5'-GGCAAATTTCCTGGTTATATCC-3' }\end{array}$ \\
\hline $\mathrm{IFN}-\gamma$ & $\begin{array}{l}\text { F: 5'-AGGCCATCAGCAACAACATAAGTG-3' } \\
\text { R: 5'-GACAGCTTTGTGCTGGATCTGTG-3' }\end{array}$ \\
\hline IL-10 & $\begin{array}{l}\text { F: 5'-GGACTTTAAGGGTTACTTGGG-3' } \\
\text { R: 5'-AGAAATCGATGACAGCGTCG-3' }\end{array}$ \\
\hline IL-4 & $\begin{array}{l}\text { F: 5'-GAACTCACTGAGAAGCTGCA-3' } \\
\text { R: 5'-GAAGTGCAGGACTGCAAGT-3' }\end{array}$ \\
\hline $\mathbb{I L}-13$ & $\begin{array}{l}\text { F: 5'-AGACCAGAAGACTTCCCTGT-3' } \\
\text { R: 5'-TCAATATCCTCTGGGTCCTG-3' }\end{array}$ \\
\hline CCR7 & $\begin{array}{l}\text { F: 5'-TGGTCATTTTCCAGGTGTGCT-3' } \\
\text { R: 5'-TACAGGGTGTAGTCCACGGT-3' }\end{array}$ \\
\hline CD163 & $\begin{array}{l}\text { F: 5'-GTAGTAGTCATTCAACCCTCAC-3' } \\
\text { R: 5'-CGGCTTACAGTTTCCTCAAG-3' }\end{array}$ \\
\hline
\end{tabular}

\section{Collagen type I expression of tendon cells}

After culture in media containing BMSC-EVs $(0,10,20 \mu \mathrm{g}$ / $\mathrm{mL}$ ) for $48 \mathrm{~h}$, tendon cells were harvested for RNA extraction. Total RNA was extracted using an RNeasy Mini Kit (Qiagen, Hilden, Germany) according to the manufacturer's instructions. cDNA was synthesized using a First-Strand Kit (Invitrogen, Carlsbad, CA). qRT-PCR was carried out with QuantiTect a SYBR Green RT-PCR Kit (Qiagen, Hilden, Germany). Gene expression assay were performed as described previously. Glyceraldehyde-3-phosphate dehydrogenase (GAPDH) was used as an internal control. Relative gene expression was calculated using the $2^{\Delta \mathrm{CT}}$ formula. Rat-specific primer for collagen type I was as follows: 5'-CCGGACTGTGAGGTTAGGAT-3' (forward) and 5'-AACCCAAAGGACCCAAATAC-3' (reverse).

\section{Cell proliferation assay}

The effects of BMSC-EVs on the proliferation of tendon cells were determined by using the CCK 8 assay. Briefly, tendon cells were seeded at $1 \times 10^{4}$ cells/well onto 96-well plates in $200 \mu \mathrm{L}$ of complete culture medium. After $24 \mathrm{~h}$ in culture, cells were treated with medium containing different concentrations of BMSC-EVs $(0,10,20 \mu \mathrm{g} / \mathrm{mL})$ for 3 days. The value of optical density was measured with a microplate reader at $450 \mathrm{~nm}$ according to the manufacturer's instructions.

\section{Statistical analysis}

All values are expressed as the mean \pm standard deviation (SD). Data were compared by an analysis of variance (ANOVA) followed by Tukey's test. Because the number of rats was small for parametric statistics, the KruskalWallis test was used to compare the results of histological analysis among groups. Statistical analyses were carried out with SPSS 11.0 statistical package. All $p$ values less than 0.05 were considered statistically significant.

\section{Results}

\section{Characterization of BMSCs and BMSCs-EVs}

Bone marrow-derived multipotent mesenchymal stromal cells were analyzed for expression of a panel of cell surface markers as shown in Fig. 2. In the FACS analysis, BMSCs were positive for mesenchymal markers CD90 (99.13\%) and CD44 (97.58\%), but negative for hematopoietic markers CD34 (0.02\%) and C11b (0.15\%). Morphology and multipotency of BMSCs were showed in Fig. 2.

Surface markers CD9, CD63, and HSP70 were presented high expression in BMSCs-EVs (Fig. 3a). Under transmission electron microscopy, BMSCs-EVs appeared as circular particles (Fig. 3c).

\section{Histological analysis}

Tendons treated with BMSC-EVs showed regularly aligned and compact collagen fibers in contrast to the disorganized and disrupted scar-like healing in tendons from fibrin and control groups (Fig. 4a, b). The fiber alignment score was significantly higher for the rats in the BMSC-EVs group compared with those in the fibrin and control groups (Fig. 4c).

\section{Effect of BMSC-EVs on macrophage polarization and inflammatory response}

We found elevated expression of CD163 as a marker of anti-inflammatory macrophages in BMSC-EVs group as compared with the other groups (Fig. 5a). Corroborating this, we found a significant increase in the mRNA expression levels of IL-4 and IL-10 (M2 macrophage stimulator) in the BMSC-EVs group as compared with the fibrin and control groups (Fig. 5b, c). However, there was no significant increase in IL-13 mRNA expression in BMSC-EVs group (Fig. 5d). Furthermore, we found a reduction in the expression in each of IFN $\gamma$, IL-1B, IL-6 (markers of M1 macrophages) in the BMSC-EVs group as compared with the fibrin and control groups (Fig. $5 \mathrm{f}-\mathrm{h}$ ).

We also investigated the in vivo inflammatory responses among the three groups. At 2 weeks, we found a significantly higher number of cells were expressing IL-10 in the BMSC-EVs group as compared with the 


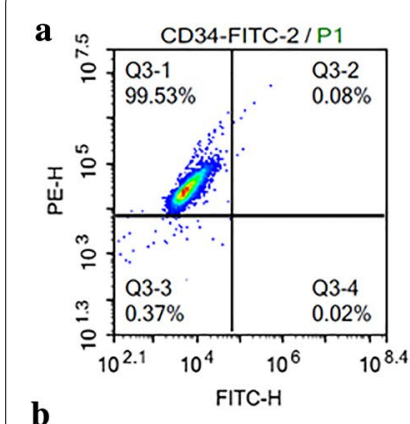

b

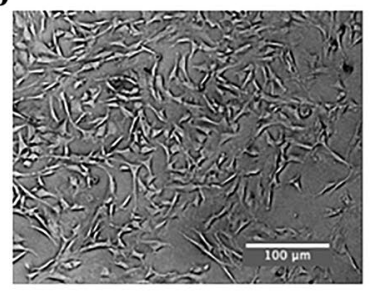

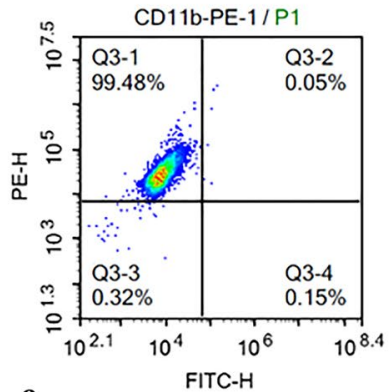

c

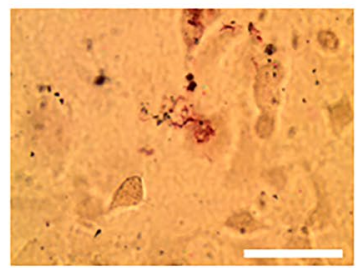

Adipogenesis
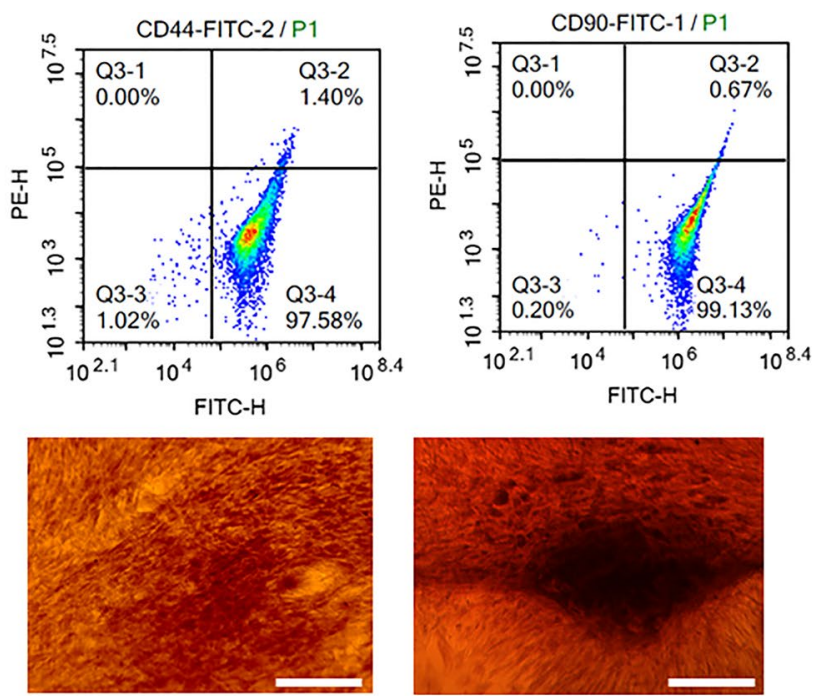

Chondrogenesis

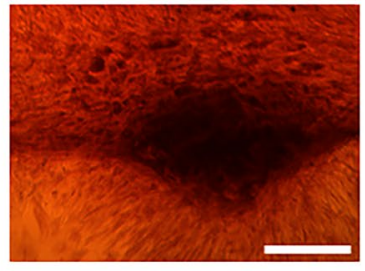

Osteogenesis

Fig. 2 Characterization of BMSCs. a FACS analysis for detection of BMSCs surface markers. b Morphology and multipotency of BMSCs. Scale bar: $100 \mu \mathrm{m}$. c The adipogenic, chondrogenic, and osteogenic differentiation potentials of BMSCs. Scale bar: $100 \mu \mathrm{m}$

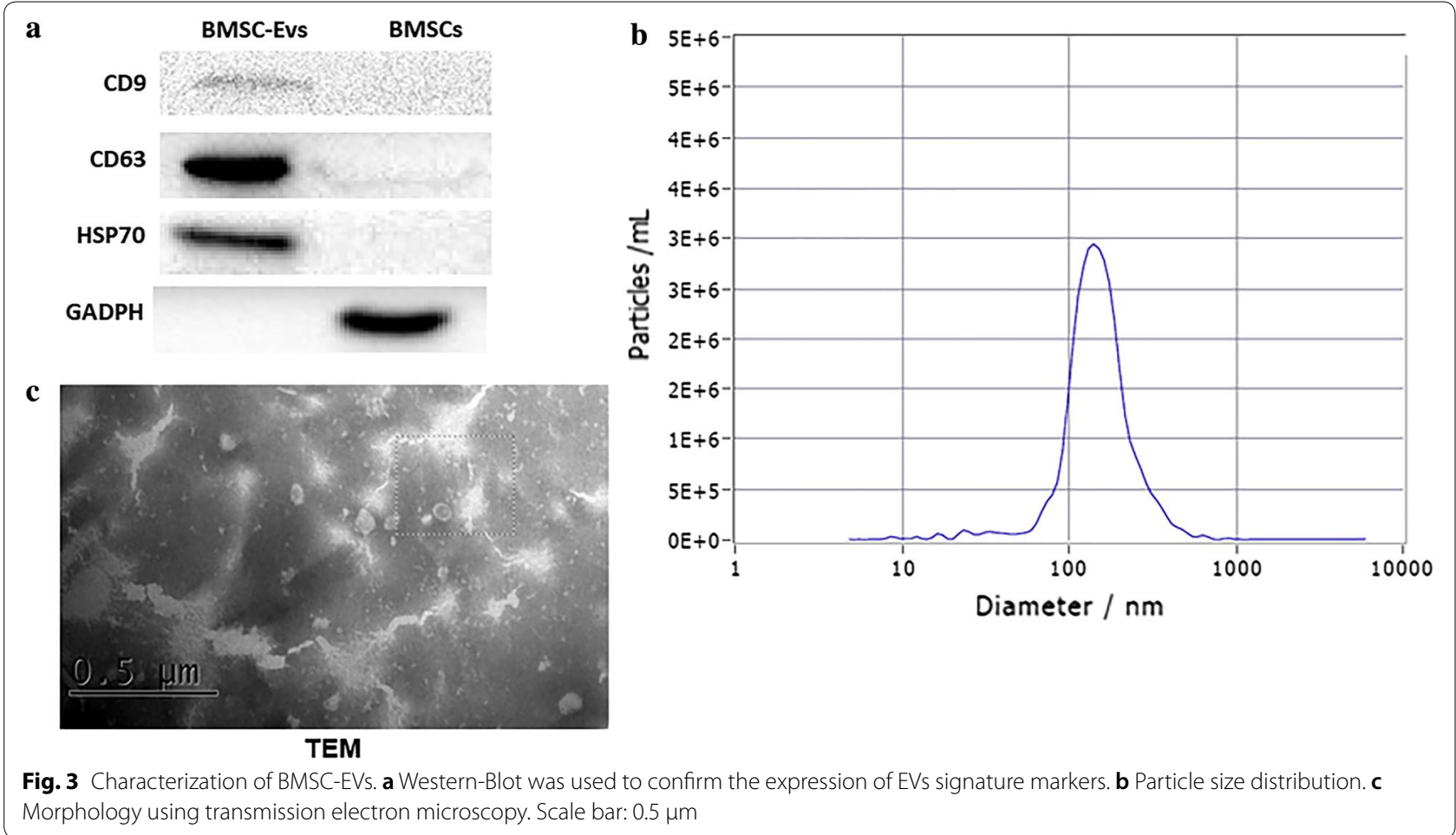

control group (Fig. 5i). Comparatively, abundant IL-6 expression was observed in the control and fibrin tendons (Fig. 5j). These results were confirmed quantitatively, with a significantly higher density of IL-10+ cells and fewer IL-6+ cells in the BMSC-EVs group than in the control group (Fig. 5k, l).

Consistent with the gene expression results, through immunostaining we identified increased numbers of 


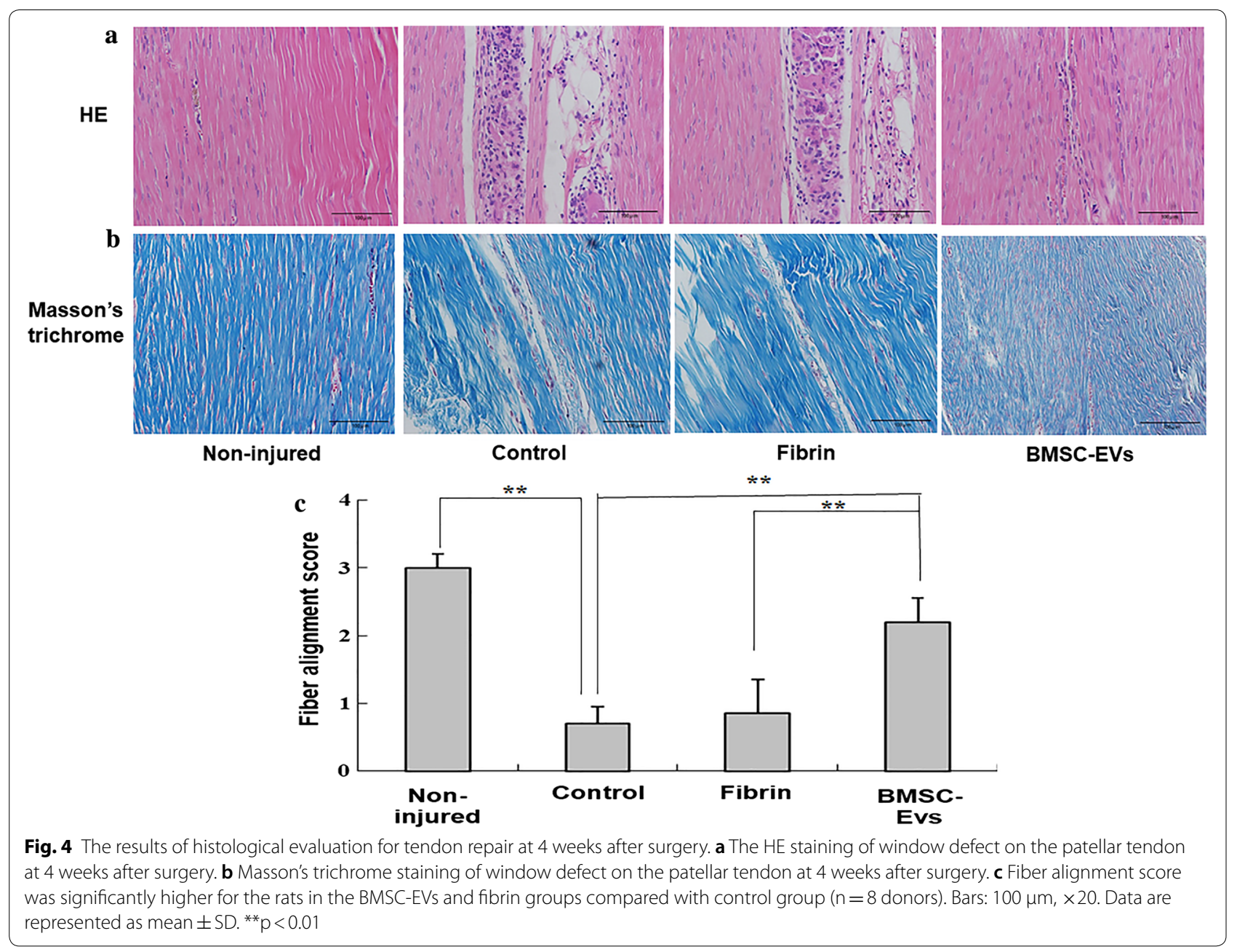

CD163+ cells at the repair site in the BMSC-EVs-treated group (Fig. 6a, c). We also found an accumulation of CCR7+ M1 macrophages in the regions of newly formed tendon tissue in the control and fibrin groups but not in the BMSC-EVs group (Fig. 6b, d), which supports the mRNA expression data.

\section{Effect of BMSC-EVs on tendon matrix formation}

We found a significantly higher expression of SCX, an early marker for tenogenic differentiation, in the BMSCEVs groups as compared with the control and fibrin groups (Fig. 7b). Likewise, the expression of TNMD, a mature marker of tenogenic differentiation, was also significantly higher in the BMSC-EVs group compared with the control group (Fig. 7c). For extracellular matrix (ECM) gene expression, COL1a1 (which encodes for type I collagen) and COL3a1 (which encodes type III collagen) were upregulated following BMSC-EVs treatment, with a relatively higher abundance of COL1a1 as compared with COL3a1 (Fig. 7a, d).

\section{Effect of BMSC-EVs on tenogenesis during tendon healing} CD146+ is a marker of tendon stem cells. At the tissue level, BMSC-EVs treatment induced an accumulation of CD146+ cells at the repair site of treated tendons (Fig. 8a, c). However, no apparent CD146 staining was observed in the control or fibrin groups, suggesting the pro-regenerative effects of BMSC-EVs in tendon repair.

\section{Effect of BMSC-EVs on tendon cell apoptosis}

M1 macrophage activation has been linked to apoptotic cell death at the tendon repair site. Therefore, we tested the potential protective effects of BMSC-EVs against apoptotic cell death during repair. In the control group, 


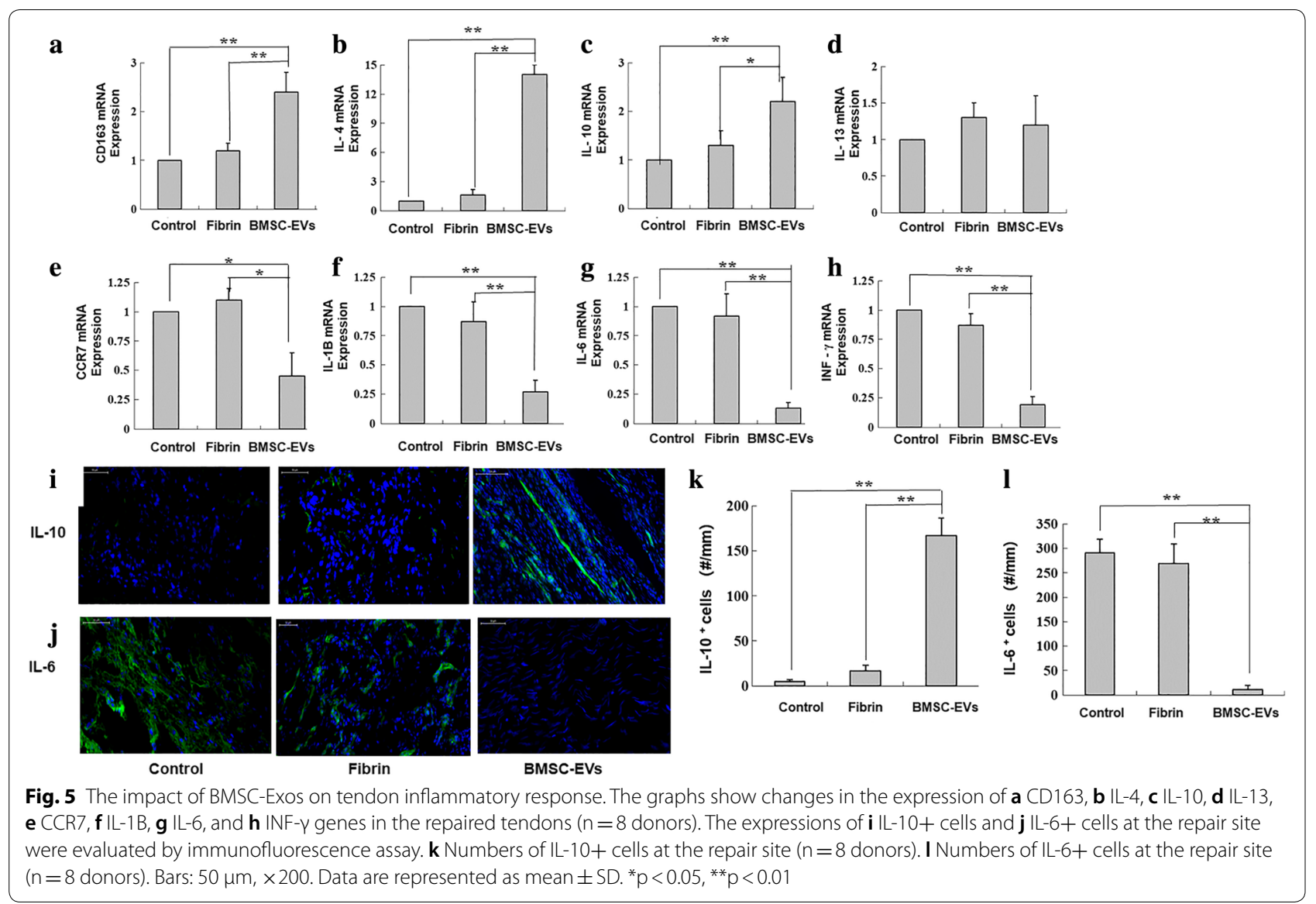

cleaved caspase 3 (a marker of apoptotic cells) signals primarily accumulated at the repair site (Fig. 8b, d). Comparatively, cleaved caspase 3 signals were reduced in tendons from the BMSC-EVs group.

\section{Effect of BMSC-EVs on tendon cell proliferation and collagen type I expression}

Cells were treated with BMSC-EVs, and viable cells were monitored by CCK- 8 assay. Tendon cells exhibited an enhanced proliferative capacity after treatment with BMSC-EVs at 10 and $20 \mu \mathrm{g} / \mathrm{mL}$ concentrations for 3 days (Fig. 9a). However, at concentration of $10 \mu \mathrm{g} / \mathrm{mL}$, the proliferation of tendon cells was not significantly different from the control. Moreover, the expression of collagen type I, a tenocyte-related gene, were increased by BMSCEVs (10 and $20 \mathrm{ng} / \mathrm{mL}$ ) (Fig. 9b), whereas no significant difference was detected at BMSC-EVs $(10 \mathrm{ng} / \mathrm{mL})$ group.

\section{Discussion}

Tendon healing progresses through three phases: inflammation, proliferation, and remodeling. The marked inflammatory response that occurs after tendon injury leads to a loss of ECM organization, the formation of scar tissue, and other degenerative events in tendons. Controlling the inflammatory environment after tendon injury is therefore a potential therapeutic target for enhanced healing $[23,24]$. Previous studies indicate that early suppression of the inflammatory response through the delivery of MSCs could promote tendon and ligament regeneration to their original state $[25,26]$. In this study, we hypothesized that BMSC-derived EVs may play a significant role in regulating inflammation and tendon healing.

We first examined changes in the tendons using $\mathrm{H} \& \mathrm{E}$ staining and Masson's trichrome staining at 4 weeks after injury. We found that the delivery of BMSC-EVs to the injury site led to a better arrangement of collagen fibers, with fibers more often oriented along the longitudinal axis of the tendon. Masson's trichrome staining further confirmed the improved tissue quality in the BMSCEVs-treated group, with an abundance of a collagen-rich ECM in the tendons as compared with those in the other groups.

In many injured tissues, proinflammatory macrophages (M1) promote ECM breakdown, inflammation, and 

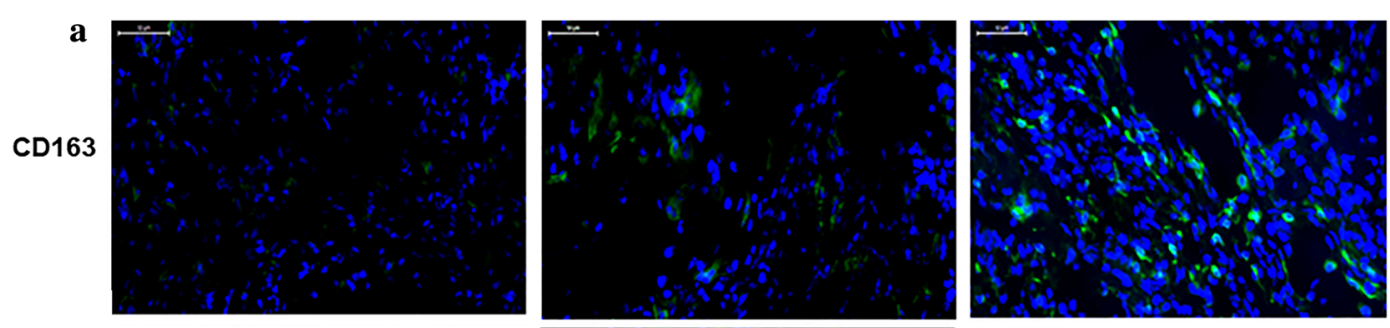

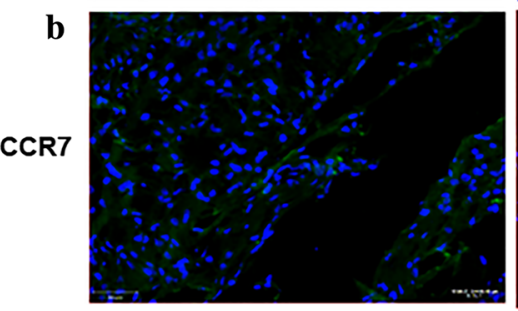

Control

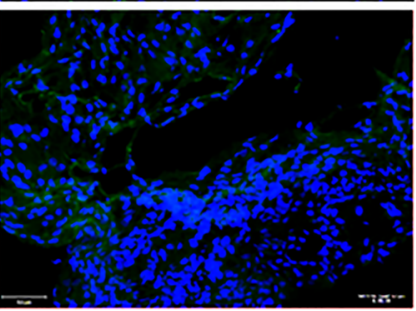

Fibrin

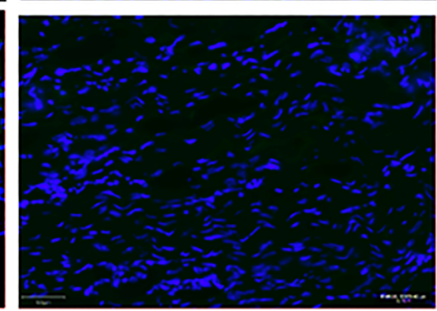

BMSC-EVs

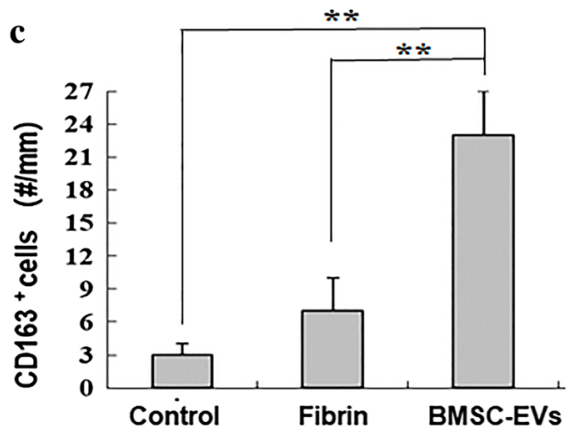

d

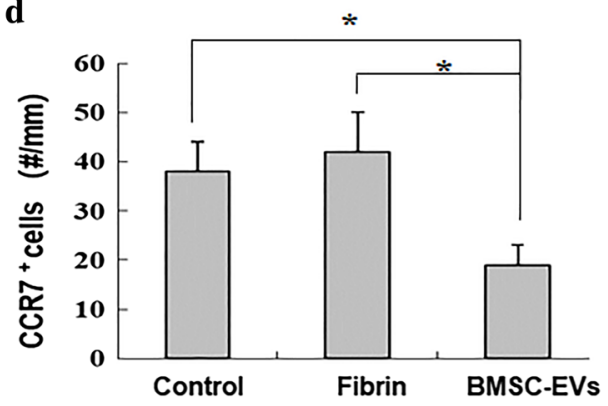

Fig. 6 The expressions of $\mathbf{a}$ CD163+ cells and $\mathbf{b}$ CCR7+ cells at the repair site were evaluated by immunofluorescence assay. $\mathbf{c}$ Numbers of CD163+ cells at the repair site $(n=8$ donors). $\mathbf{d}$ Numbers of CCR7+ cells at the repair site $(n=8$ donors). Bars: $50 \mu m, \times 200$. Data are represented as mean $\pm S D$. ${ }^{*} p<0.05,{ }^{* *} p<0.01$

apoptosis [27-29]. However, anti-inflammatory macrophages (M2) often coordinate ECM deposition and tissue repair. Here, we showed a significant reduction in the proportion of CCR7+ M1 cells and an increase in CD163+ M2 macrophages in tendons from the BMSCEVs group. Furthermore, we found elevated expression of M2 stimulator genes IL-4 and IL-10, and lower levels of inflammatory cytokines (IFN $\gamma$, IL-1B, and IL-6). Based on these findings, BMSC-EVs treatment may improve tendon healing by influencing the balance of macrophages and their associated cytokines toward an anti-inflammatory environment. IL13 is a proinflammatory cytokine produced by activated $\mathrm{T}$ cells, which can promote the secretion of other inflammatory cytokines and the expression of adhesion molecules. It can regulate collagen stability of fibroblasts. Chronic inflammation induced by IL-13 contributes to the pathogenic scar and fibrosis process by recruiting fibrocytes, which in turn leads to the deposition of excessive ECM that destroy the healing tissue architecture [30]. There was no significant increasement in IL-13 mRNA expression in healing tendon tissue subsequent to BMSCs-EVs treatment in our study, suggesting that BMSCs-EVs may play an important role in improving tendon repair and remodeling after injury.

Based on the changes in the expression of matrix genes involved in tendon healing, our results suggest that BMSC-EVs treatment has a positive effect on repair. We found increased expression of collagen I and III mRNA in healing tendons treated with BMSC-EVs as compared with injured tendons in the control and fibrin groups. Collagen $I$ is the predominant type of collagen in normal tendon tissue, responsible for providing structural and mechanical properties [20]. Type III collagen has been linked to scar formation and inferior tendon mechanical properties after injury [31]. It has been suggested that an optimized collagen type I/III ratio might account for the quality of matrix organization in tendon healing [32]. We found that the relative abundance of COL1a1 was approximately twofold higher than that of COL3a1 in tendons from the BMSC-EVs group. This finding suggests that BMSC-EVs might help to promote the synthesis of ECM components suitable for repair. 

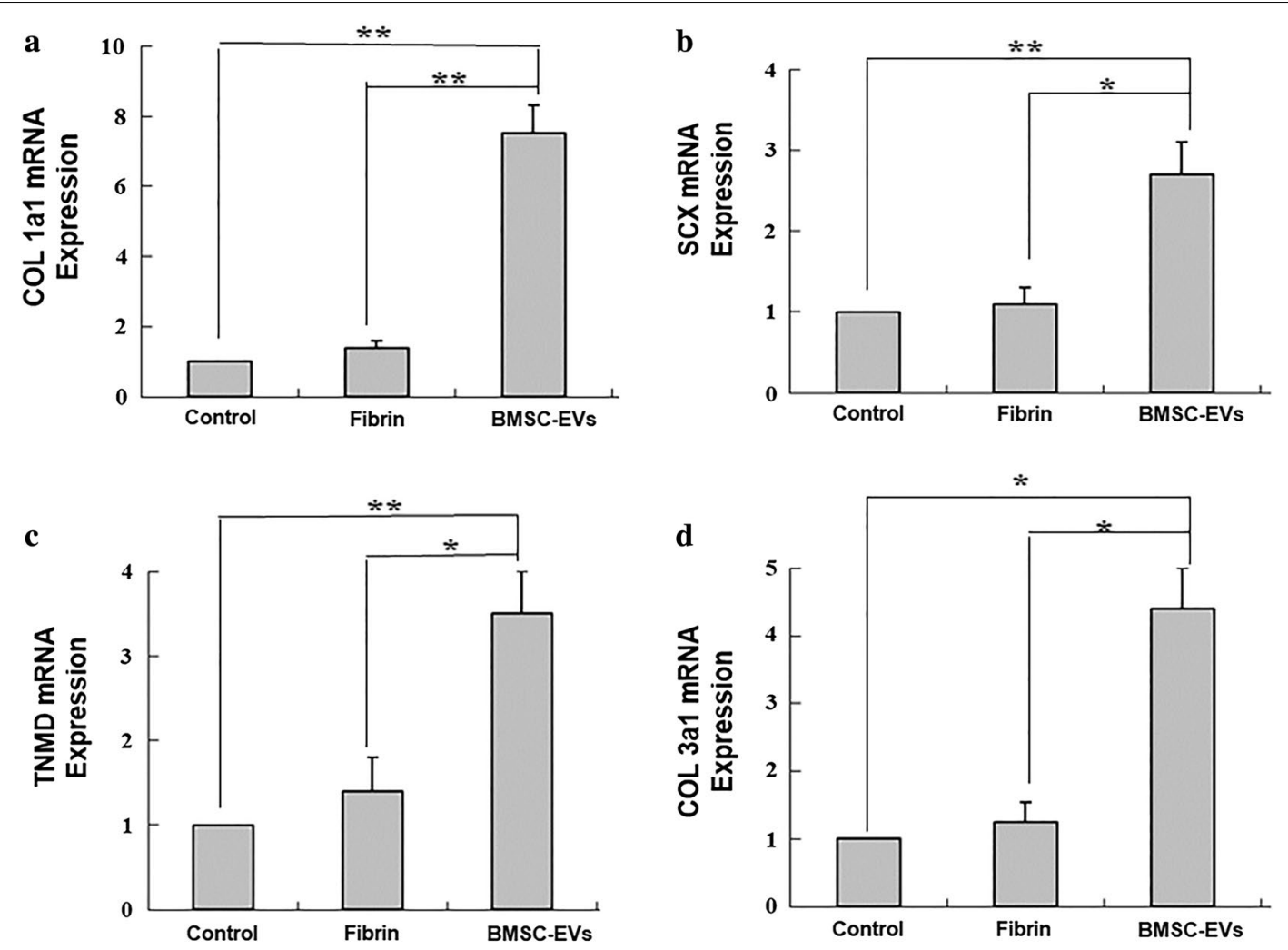

Fig. 7 The expression of tenocyte-related genes, a COL1a1, b SCX, c TNMD, and $\mathbf{d}$ COL3a1 in tendons 14 days after repair $(\mathrm{n}=8 \mathrm{donors})$. Data are represented as mean $\pm S D$. ${ }^{*} p<0.05,{ }^{* *} p<0.01$

We also examined the expression of genes related to tenocyte differentiation. SCX is a basic helix-loop-helix transcription factor involved in tendon development, the deletion of which dramatically disrupts tendon differentiation. SCX is also a distinct marker of tendon progenitors and differentiated cells [33]. In our study, SCX expression was elevated in tendons from the BMSC-EVs group at 2 weeks as compared with the other groups. TNMD is another transcription factor involved in tendon maturation. In the present study, we found a significant increase in TNMD in the BMSC-EVs group over that in the other groups. These results suggest that BMSC-EVs may promote tendon healing by regulating tenogenesis.

Previous studies have revealed that apoptosis plays an important role in regulating tendon regeneration during wound healing [34-36], with higher numbers of apoptotic cells altering the composition of the tendon matrix during repair. Efficient tendon healing should inhibit apoptosis. We found fewer apoptotic cells in the tendons treated with BMSC-EVs, which may suggest that BMSCEVs can help to prevent the accumulation of apoptotic cells and the subsequent scar tissues that form during early tendon healing.
Previous studies have shown that a reduction in the presence of M2 macrophages and the loss of TNMD expression are associated with fewer CD146-positive cells and more erroneous matrix deposition at the repair site $[8,36]$. Furthermore, enriching for CD146-positive cells can lead to improved tendon healing outcomes $[6,8,37]$. Therefore, as a final test, we examined the expression of CD146 in the healing tendons. We found higher proportions of CD146+ cells in the BMSC-EVs group, which suggests the potential for this approach to enhance tendon regeneration. Our findings also demonstrate that regulating the proportion of CD146+ tendon cells may provide an important foundation for the development of a new strategy for tendon healing by encouraging the regenerative capacity of tendon-resident stem/progenitor cells.

Fibrin sealant has been used extensively in all kinds of surgery and research. In this work, an integrated repair was proved effectively by fibrin glue consist of BMSCEVs in a rat tendon injury model. It could form a stable fibrin polymer after applied to injury site and the window defects of injured tendons were closed with fibrin sealant. Mixing BMSC-EVs in fibrin glue is easy to deliver locally and safe. This method may be a feasible approach 

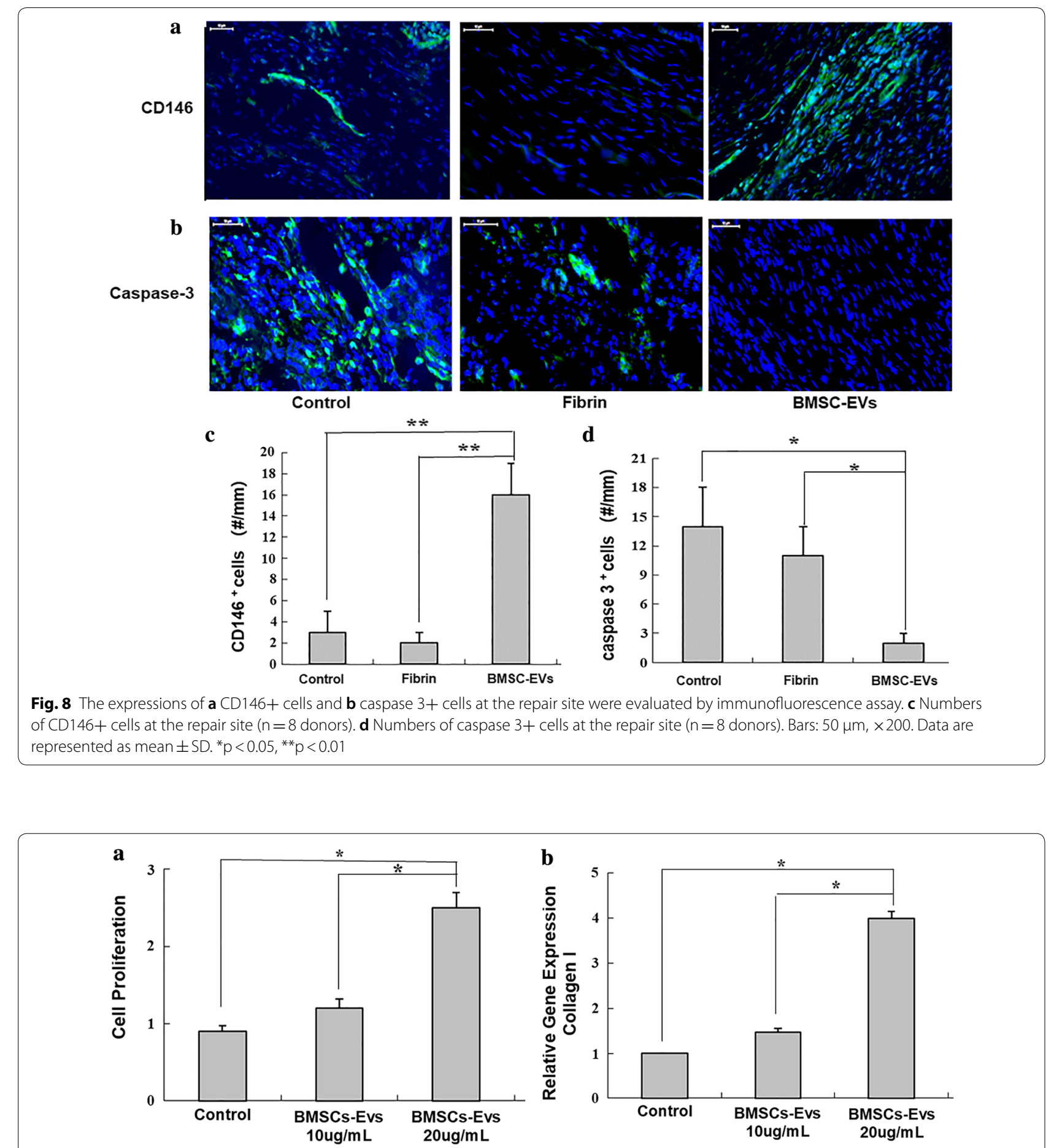

Fig. 9 Tendon cell proliferation and collagen type I expression. a Treatment of tendon cells with BMSC-EVs $(20 \mu \mathrm{g} / \mathrm{mL})$ increased cell proliferation significantly. b The expression of collagen type l, was increased by BMSC-EVs $(20 \mathrm{ng} / \mathrm{mL})$. Data are represented as mean $\pm S D$. ${ }^{*} \mathrm{p}<0.05$

for repairing tendon injury in the clinic. Future studies should evaluate the physiological and mechanical properties of the fibrin glue consist of BMSC-EVs for longer period $[38,39]$.
There were several limitations to the current study. First, the follow-up time points of 2 and 4 weeks in this study may not be long enough for the final analysis, and there may be actual tissue healing status at later time. 
Study with a longer evaluation period may be needed in future. Second, EVs contain a set of bioactive and tissue trophic molecules such as proteins, nucleic acids, and lipid molecules. Further studies investigating protein or nucleic acids, and which molecular content of the EVs modulate tendon healing are needed to reinforce the results of the current study.

\section{Conclusions}

In summary, we demonstrate that EVs derived from BMSCs can help to improve the quality of tendon healing by modulating macrophage phenotypes, promoting an antiinflammatory environment, and encouraging the regenerative capacity of tendon-resident stem/progenitor cells. The beneficial effects of BMSC-EVs delivery in tendon healing may offer a new avenue for promoting tendon regeneration. Future studies will explore the potential for this approach to enhance the functional outcomes after tendon repair.

\begin{abstract}
Abbreviations
a-MEM: alpha-modified Eagle's medium; BCA: bicinchoninic acid; BMSC-EVs: bone marrow mesenchymal stem cells; CCR7: C-C chemokine receptor type 7; COL: collagen; FBS: fetal bovine serum; GAPDH: glyceraldehyde-3-phosphate dehydrogenase; H\&E: hematoxylin-eosin; IFNy: interferon gamma; IL: interleukin; MSCs: mesenchymal stem cells; PBS: phosphate-buffered saline; SCX: scleraxis; SD: standard deviation; TNMD: tenomodulin; TSCs: tendon stem/ progenitor cells.
\end{abstract}

\section{Acknowledgements}

We thank Dr. Shulong Yang for his suggestions to revise the manuscript.

\section{Authors' contributions}

ZZS and QW carried out the main part of the studies and drafted the manuscript. DPJ participated in the design of the study and performed the statistical analysis. All authors read and approved the final manuscript.

\section{Funding}

This project was supported by Grants from the National Natural Science Foundation of China (No. 81472085) and the Natural Science Foundation of Shanghai (No. 19ZR1432800).

\section{Availability of data and materials}

All data generated or analyzed during this study are included in this manuscript.

\section{Ethics approval and consent to participate}

The study was approved by the Ethics Committee of Shanghai Children's

Medical Center, Shanghai Jiao Tong University School of Medicine (China).

\section{Consent for publication}

Not applicable.

\section{Competing interests}

The authors declare that they have no competing interests.

Received: 7 April 2019 Accepted: 21 June 2019

Published online: 25 June 2019

\section{References}

1. Dams OC, van den Akker-Scheek I, Diercks RL, Wendt KW, Zwerver J, Reininga IHF. Surveying the management of Achilles tendon ruptures in the
Netherlands: lack of consensus and need for treatment guidelines. Knee Surg Sports Traumatol Arthrosc. 2018. https://doi.org/10.1007/s0016 7-018-5049-5.

2. Morita W, Dakin SG, Snelling SJB, Carr AJ. Cytokines in tendon disease: a systematic review. Bone Joint Res. 2017;6:656-64.

3. Hammerman M, Blomgran P, Dansac A, Eliasson P, Aspenberg P. Different gene response to mechanical loading during early and late phases of rat Achilles tendon healing. J Appl Physiol. 1985;2017(123):800-15.

4. Thankam FG, Roesch ZK, Dilisio MF, Radwan MM, Kovilam A, Gross RM, Agrawal DK. Association of inflammatory responses and ECM disorganization with HMGB1 upregulation and NLRP3 inflammasome activation in the injured rotator cuff tendon. Sci Rep. 2018;8:8918.

5. Ackermann PW, Domeij-Arverud E, Leclerc P, Amoudrouz P, Nader GA. Anti-inflammatory cytokine profile in early human tendon repair. Knee Surg Sports Traumatol Arthrosc. 2013;21:1801-6.

6. Shen H, Jayaram R, Yoneda S, Linderman SW, Sakiyama-Elbert SE, Xia Y, Gelberman RH, Thomopoulos S. The effect of adipose-derived stem cell sheets and CTGF on early flexor tendon healing in a canine model. Sci Rep. 2018;8:11078.

7. Zhou Y, Zhang J, Wu H, Hogan MV, Wang JH. The differential effects of leukocyte-containing and pure platelet-rich plasma (PRP) on tendon stem/progenitor cells-implications of PRP application for the clinical treatment of tendon injuries. Stem Cell Res Ther. 2015;6:173.

8. Gelberman RH, Linderman SW, Jayaram R, Dikina AD, Sakiyama-Elbert S, Alsberg E, Thomopoulos S, Shen H. Combined administration of ASCS and BMP-12 promotes an M2 macrophage phenotype and enhances tendon healing. Clin Orthop Relat Res. 2017:475:2318-31.

9. Tarafder S, Chen E, Jun Y, Kao K, Sim KH, Back J, Lee FY, Lee CH. Tendon stem/progenitor cells regulate inflammation in tendon healing via JNK and STAT3 signaling. FASEB J. 2017;31:3991-8.

10. Wang LL, Yin XF, Chu XC, Zhang YB, Gong XN. Platelet-derived growth factor subunit $B$ is required for tendon-bone healing using bone marrowderived mesenchymal stem cells after rotator cuff repair in rats. J Cell Biochem. 2018. https://doi.org/10.1002/jcb.27143.

11. Harris MT, Butler DL, Boivin GP, Florer JB, Schantz EJ, Wenstrup RJ. Mesenchymal stem cells used for rabbit tendon repair can form ectopic bone and express alkaline phosphatase activity in constructs. J Orthop Res. 2004;22:998-1003.

12. Blum B, Bar-Nur O, Golan-Lev T, Benvenisty N. The antiapoptotic gene survivin contributes to teratoma formation by human embryonic stem cells. Nat Biotechnol. 2009;27:281-7.

13. Pelizzo G, Avanzini MA, Icaro Cornaglia A, De Silvestri A, Mantelli M, Travaglino P, Croce S, Romano P, Avolio L, lacob G, Dominici M, Calcaterra V. Extracellular vesicles derived from mesenchymal cells: perspective treatment for cutaneous wound healing in pediatrics. Regen Med. 2018;13:385-94.

14. Zhang J, Guan J, Niu X, Hu G, Guo S, Li Q, Xie Z, Zhang C, Wang Y. Exosomes released from human induced pluripotent stem cells-derived MSCs facilitate cutaneous wound healing by promoting collagen synthesis and angiogenesis. J Transl Med. 2015;13:49.

15. Murphy KC, Whitehead J, Zhou D, Ho SS, Leach JK. Engineering fibrin hydrogels to promote the wound healing potential of mesenchymal stem cell spheroids. Acta Biomater. 2017;64:176-86.

16. Ju C, Shen Y, Ma G, Liu Y, Cai J, Kim IM, Weintraub NL, Liu N, Tang Y. Transplantation of cardiac mesenchymal stem cell-derived exosomes promotes repair in ischemic myocardium. J Cardiovasc Transl Res. 2018. https://doi.org/10.1007/s12265-018-9822-0.

17. Bai Y, Han YD, Yan XL, Ren J, Zeng Q, Li XD, Pei XT, Han Y. Adipose mesenchymal stem cell-derived exosomes stimulated by hydrogen peroxide enhanced skin flap recovery in ischemia-reperfusion injury. Biochem Biophys Res Commun. 2018;500:310-7.

18. Yang J, Liu XX, Fan H, Tang Q, Shou ZX, Zuo DM, Zou Z, Xu M, Chen QY, Peng Y, Deng SJ, Liu YJ. Extracellular vesicles derived from bone marrow mesenchymal stem cells protect against experimental colitis via attenuating colon inflammation, oxidative stress and apoptosis. PLoS ONE. 2015;10:e0140551.

19. Xu B, Yang M, Li Z, Zhang Y, Jiang Z, Guan S, Jiang D. Thymosin $\beta 4$ enhances the healing of medial collateral ligament injury in rat. Regul Pept. 2013;184:1-5.

20. Wen Q, Zhou C, Luo W, Zhou M, Ma L. Pro-osteogenic effects of fibrin glue in treatment of avascular necrosis of the femoral head in vivo by 
hepatocyte growth factor-transgenic mesenchymal stem cells. J Transl Med. 2014;12:114.

21. Jiang $D$, Gao $P$, Lin $H$, Geng $H$. Curcumin improves tendon healing in rats: a histological, biochemical, and functional evaluation. Connect Tissue Res. 2016;57:20-7.

22. Vinhas A, Rodrigues MT, Gomes ME. Exploring stem cells and inflammation in tendon repair and regeneration. Adv Exp Med Biol. 2018;1089:37-46.

23. Chamberlain CS, Clements AEB, Kink JA, Choi U, Baer GS, Halanski MA, Hematti P, Vanderby R. Extracellular vesicle-educated macrophages promote early achilles tendon healing. Stem Cells. 2019;37:652-62.

24. Al-Sadi O, Schulze-Tanzil G, Kohl B, Lohan A, Lemke M, Ertel W, John T. Tenocytes, pro-inflammatory cytokines and leukocytes: a relationship? Muscles Ligaments Tendons J. 2012;1:68-76.

25. Noack S, Seiffart V, Willbold E, Laggies S, Winkel A, Shahab-Osterloh S, Flörkemeier T, Hertwig F, Steinhoff C, Nuber UA, Gross G, Hoffmann A. Periostin secreted by mesenchymal stem cells supports tendon formation in an ectopic mouse model. Stem Cells Dev. 2014;23:1844-57.

26. Chamberlain CS, Saether EE, Aktas E, Vanderby R. Mesenchymal stem cell therapy on tendon/ligament healing. J Cytokine Biol. 2017;2:112.

27. Dai M, Sui B, Xue Y, Liu X, Sun J. Cartilage repair in degenerative osteoarthritis mediated by squid type II collagen via immunomodulating activation of M2 macrophages, inhibiting apoptosis and hypertrophy of chondrocytes. Biomaterials. 2018;180:91-103.

28. Laplante P, Brillant-Marquis F, Brissette MJ, Joannette-Pilon B, Cayrol R, Kokta V, Cailhier JF. MFG-E8 reprogramming of macrophages promotes wound healing by increased bFGF production and fibroblast functions. J Invest Dermatol. 2017;137:2005-13.

29. Klinkert K, Whelan D, Clover AJP, Leblond AL, Kumar AHS, Caplice NM. Selective M2 macrophage depletion leads to prolonged inflammation in surgical wounds. Eur Surg Res. 2017:58:109-20,

30. Oh MH, Oh SY, Yu J, Myers AC, Leonard WJ, Liu YJ, Zhu Z, Zheng T. IL-13 induces skin fibrosis in atopic dermatitis by thymic stromal lymphopoietin. J Immunol. 2011;186:7232-42.

31. Juneja SC, Schwarz EM, O'Keefe RJ, Awad HA. Cellular and molecular factors in flexor tendon repair and adhesions: a histological and gene expression analysis. Connect Tissue Res. 2013;54:218-26.
32. Matsumoto F, Trudel G, Uhthoff HK. High collagen type I and low collagen type III levels in knee joint contracture: an immunohistochemical study with histological correlate. Acta Orthop Scand. 2002;73:335-43.

33. Schweitzer R, Chyung JH, Murtaugh LC, Brent AE, Rosen V, Olson EN, Lassar A, Tabin CJ. Analysis of the tendon cell fate using Scleraxis, a specific marker for tendons and ligaments. Development. 2001;128:3855-66.

34. Ramírez JP, Bonati-Richardson F, García MP, Hidalgo C, Stoore C, Liendo R, Soza F, Landerer E, Paredes R. Intra-articular treatment with corticosteroids increases apoptosis in human rotator cuff tears. Connect Tissue Res. 2019;60:283-90.

35. Bell R, Robles-Harris MA, Anderson M, Laudier D, Schaffler MB, Flatow EL, Andarawis-Puri N. Inhibition of apoptosis exacerbates fatigue-damage tendon injuries in an in vivo rat model. Eur Cell Mater. 2018;36:44-56.

36. Lin D, Alberton P, Caceres MD, Volkmer E, Schieker M, Docheva D. Tenomodulin is essential for prevention of adipocyte accumulation and fibrovascular scar formation during early tendon healing. Cell Death Dis. 2017;8:e3116.

37. Lee CH, Lee FY, Tarafder S, Kao K, Jun Y, Yang G, Mao JJ. Harnessing endogenous stem/progenitor cells for tendon regeneration. J Clin Invest. 2015;125:2690-701.

38. Lötvall J, Hill AF, Hochberg F, Buzás El, Di Vizio D, Gardiner C, Gho YS, Kurochkin IV, Mathivanan S, Quesenberry P, Sahoo S, Tahara H, Wauben MH, Witwer KW, Théry C. Minimal experimental requirements for definition of extracellular vesicles and their functions: a position statement from the International Society for Extracellular Vesicles. J Extracell Vesicles. 2014;3:26913.

39. Gould SJ, Raposo G. As we wait: coping with an imperfect nomenclature for extracellular vesicles. J Extracell Vesicles. 2013;2:20389 (eCollection 2013).

\section{Publisher's Note}

Springer Nature remains neutral with regard to jurisdictional claims in published maps and institutional affiliations.

Ready to submit your research? Choose BMC and benefit from:

- fast, convenient online submission

- thorough peer review by experienced researchers in your field

- rapid publication on acceptance

- support for research data, including large and complex data types

- gold Open Access which fosters wider collaboration and increased citations

- maximum visibility for your research: over 100M website views per year

At BMC, research is always in progress.

Learn more biomedcentral.com/submissions 